\title{
ON THE NUMBER OF CYCLIC SUBGROUPS OF A FINITE GROUP
}

\author{
MARTINO GARONZI AND IGOR LIMA
}

\begin{abstract}
Let $G$ be a finite group and let $c(G)$ be the number of cyclic subgroups of $G$. We study the function $\alpha(G)=c(G) /|G|$. We explore its basic properties and we point out a connection with the probability of commutation. For many families $\mathscr{F}$ of groups we characterize the groups $G \in \mathscr{F}$ for which $\alpha(G)$ is maximal and we classify the groups $G$ for which $\alpha(G)>3 / 4$. We also study the number of cyclic subgroups of a direct power of a given group deducing an asymptotic result and we characterize the equality $\alpha(G)=\alpha(G / N)$ when $G / N$ is a symmetric group.
\end{abstract}

\section{INTRODUCTION}

In this paper all the groups we consider are finite. Let $c(G)$ be the number of cyclic subgroups of a group $G$ and $\alpha(G):=c(G) /|G|$. It is clear that $0<\alpha(G) \leq 1$. Observe that every cyclic subgroup $\langle x\rangle$ of $G$ has $\varphi(o(x))$ generators, where $\varphi$ is Euler's totient function and $o(x)$ denotes the order of the element $x$, hence

$$
c(G)=\sum_{x \in G} \frac{1}{\varphi(o(x))} .
$$

On a computational level this formula is probably best for computing $c(G)$ for arbitrary $G$, and it is what we employed to work out small groups using [3]. For $d$ a divisor of $|G|=n$ let $B_{G}(d)$ be the number of elements $x \in G$ such that $x^{d}=1$. Denote by $\mu$ the standard Möbius function. In 4 another formula is given (from Lemma 7 (2) choosing $A(d)$ the number of elements of $G$ of order $d, B(d)=B_{G}(d)$ and $(r, s)=(1,0))$, which is an easy application of the Möbius inversion formula, the following:

$$
c(G)=\sum_{d \mid n}\left(\sum_{i \mid n / d} \frac{\mu(i)}{\varphi(d i)}\right) B_{G}(d) .
$$

Using this formula in [4, Corollary 13] it was shown that ciclicity can be detected by the number of cyclic subgroups, more precisely that if $|G|=n$ then $c(G) \geq c\left(C_{n}\right)$ with equality if and only if $G \cong C_{n}$.

There is a connection between $\alpha(G)$ and the so-called "commuting probability" of $G$, denoted by $\operatorname{cp}(G)$, that is the probability that two random elements of $G$ commute (studied extensively in $[5$, which we crucially employ in our study). More specifically we prove that if $\alpha(G) \geq 1 / 2$ then $\operatorname{cp}(G) \geq(2 \alpha(G)-1)^{2}$. This implies that a group with many cyclic subgroups has big solvable radical and, if it is already solvable, it has big Fitting subgroup (see Section 6 for the details). 
It is not hard to show that $\alpha(G) \leq \alpha(G / N)$ whenever $N$ is a normal subgroup of $G$, and if equality holds then $N$ must be an elementary abelian 2-group. It is an interesting question to ask what can we say about $G$ if $\alpha(G)=\alpha(G / N)$ given some information on $G / N$. In this paper we characterize equality $\alpha(G)=\alpha(G / N)$ when $G / N$ is a symmetric group (Theorem 1).

Given a family $\mathscr{F}$ of groups define

$$
\alpha_{\mathscr{F}}:=\max \{\alpha(G): G \in \mathscr{F}\}, \quad m \mathscr{F}:=\left\{G \in \mathscr{F}: \alpha(G)=\alpha_{\mathscr{F}}\right\} .
$$

We are interested in computing $\alpha_{\mathscr{F}}$ and $m \mathscr{F}$ for various families $\mathscr{F}$. In this paper we prove the following results.

(1) If $\mathscr{F}$ is the family of all finite groups then $\alpha \mathscr{F}=1$ and $m \mathscr{F}$ is the family of elementary abelian 2-groups (by 2.2).

(2) If $\mathscr{F}$ is the family of non-abelian groups then

$$
\alpha_{\mathscr{F}}=7 / 8=\alpha\left(D_{8}\right)
$$

and $m \mathscr{F}$ is the family of groups of the form $C_{2}{ }^{n} \times D_{8}$ for some $n \geq 0$ (by Corollary 2).

(3) If $\mathscr{F}$ is the family of non-nilpotent groups then

$$
\alpha_{\mathscr{F}}=5 / 6=\alpha\left(S_{3}\right)
$$

and $m \mathscr{F}$ is the family of groups of the form $C_{2}{ }^{n} \times S_{3}$ for some $n \geq 0$ (by Corollary 2).

(4) If $\mathscr{F}$ is the family of non-solvable groups then

$$
\alpha_{\mathscr{F}}=67 / 120=\alpha\left(S_{5}\right)
$$

and $m \mathscr{F}$ is the family of groups of the form $C_{2}{ }^{n} \times S_{5}$ for some $n \geq 0$ (Theorem 4).

(5) If $\mathscr{F}$ is the family of non-supersolvable groups then

$$
\alpha \mathscr{F}=17 / 24=\alpha\left(S_{4}\right)
$$

and $m \mathscr{F}$ is the family of groups of the form $C_{2}{ }^{n} \times S_{4}$ for some $n \geq 0$ (Theorem 6).

(6) If $p$ is an odd prime and $\mathscr{F}$ is the family of non-trivial groups of order divisible only by primes at least $p$ then

$$
\alpha_{\mathscr{F}}=2 / p=\alpha\left(C_{p}\right)
$$

and $m \mathscr{F}=\left\{C_{p}\right\}$ (Proposition 1).

(7) If $p$ is an odd prime and $\mathscr{F}$ is the family of groups $G$ with $C_{p}$ as an epimorphic image then

$$
\alpha_{\mathscr{F}}=2 / p=\alpha\left(C_{p}\right)
$$


and $m \mathscr{F}$ is the family of groups which are the direct product of an elementary abelian 2-group and a Frobenius group with 2-elementary abelian Frobenius kernel and Frobenius complements of order $p$ (Proposition 2).

We also classify the groups $G$ with $\alpha(G)>3 / 4$ (Theorem [5), proving in particular that $3 / 4$ is the largest non-trivial accumulation point of the set of numbers of the form $\alpha(G)$. An easy consequence of this (Corollary 3) is that if $G$ is not an elementary abelian 2-group and it has $|G|-n$ cyclic subgroups then $|G| \leq 8 n$. This extends and generalizes the results in [11, as we show right after the corollary. We also give a formula for $\alpha(G)$ when $G$ is a nilpotent group (Theorem 21) and we study $\alpha$ of a direct power (Theorem 3) proving that $G^{n}$ has roughly $\left|G^{n}\right| / \varphi(\exp (G))$ cyclic subgroups.

\section{BASIC PROPERTIES OF $\alpha$}

In this section we prove some basic properties of the function $\alpha$.

2.1. If $A$ and $B$ are finite groups of coprime orders then $c(A \times B)=c(A) c(B)$ and hence $\alpha(A \times B)=\alpha(A) \alpha(B)$. The proof of this is straightforward.

2.2. Let $I(G)$ denote the number of elements $g \in G$ such that $g^{2}=1$. Then

$$
\alpha(G) \leq \frac{1}{2}+\frac{I(G)}{2|G|}, \quad \frac{I(G)}{|G|} \geq 2 \alpha(G)-1 .
$$

In particular $\alpha(G)=1$ if and only if $G$ is an elementary abelian 2-group.

Proof. If $g \in G$ then $g^{2}=1$ if and only if $\varphi(o(g))=1$, so

$$
c(G)=\sum_{x \in G} \frac{1}{\varphi(o(x))} \leq I(G)+\frac{1}{2}(|G|-I(G))=\frac{1}{2}(I(G)+|G|) .
$$

This implies the result.

2.3. If $N \unlhd G$ then $\alpha(G) \leq \alpha(G / N)$. Moreover $\alpha(G)=\alpha(G / N)$ if and only if $\varphi(o(g))=\varphi(o(g N))$ for every $g \in G$, where $o(g N)$ denotes the order of the element $g N$ in the group $G / N$.

Proof. If $a$ divides $b$ then $\varphi(a) \leq \varphi(b)$, therefore

$$
c(G / N)=\sum_{g N \in G / N} \frac{1}{\varphi(o(g N))}=\sum_{g \in G} \frac{1}{|N| \varphi(o(g N))} \geq \sum_{g \in G} \frac{1}{|N| \varphi(o(g))}=\frac{c(G)}{|N|} .
$$

This implies the result.

2.4. If $\alpha(G)=\alpha(G / N)$ then $N$ is an elementary abelian 2-group.

Proof. If $n \in N$ then applying 2.3 we have $\varphi(o(n))=\varphi(o(n N))=\varphi(o(N))=$ $\varphi(1)=1$ so $n^{2}=1$.

2.5. If $G$ is any finite group then $\alpha(G)=\alpha\left(G \times C_{2}{ }^{n}\right)$ for all $n \geq 0$.

Proof. Choosing $N=\{1\} \times{C_{2}}^{n}$ gives $\varphi(o(x))=\varphi(o(x N))$ for all $x \in G \times C_{2}{ }^{n}$. The result follows from 2.3 . 
2.6. If $\alpha(G)=\alpha(G / N)$ and $L \unlhd G, L \subseteq N$ then $\alpha(G)=\alpha(G / L)$.

Proof. Since $G / N$ is a quotient of $G / L$ we have $\alpha(G / N)=\alpha(G) \leq \alpha(G / L) \leq$ $\alpha(G / N)$ by 2.3 and the result follows.

2.7. If $\alpha(G)=\alpha(G / N)$ and $K \leq G$ then $\alpha(K)=\alpha(K / K \cap N)$.

Proof. Let $R:=K \cap N$. By 2.3, is enough to show that if $x \in K$ then $o(x R)=o(x N)$ (because then $\varphi(o(x R))=\varphi(o(x N))=\varphi(o(x)))$. Let $a=o(x R)$ and $b=o(x N)$. Since $R \subseteq N$ we have $x^{a} \in N$ so $b \leq a$. On the other hand $x^{b} \in K \cap N=R$ so $a \leq b$. Therefore $a=b$.

2.8. Suppose $\alpha(G)=\alpha(G / N)$. If $a \in G$ has order 2 modulo $N$ then $a$ centralizes $N$, in particular if $G / N$ can be generated by elements of order 2 then $N \subseteq Z(G)$.

Proof. We have $\varphi(o(a))=\varphi(o(a N))=\varphi(2)=1$ by 2.3, and $a$ has order 2 modulo $N$, so $o(a)=2$. If $n \in N$ then $\varphi(o(a n))=\varphi(o(a n N))=\varphi(o(a N))=1$ so $(a n)^{2}=1$. This together with $a^{2}=n^{2}=1$ (by 2.4) implies $a n=n a$. Recalling that $N$ is abelian (by 2.4) we deduce that if $G / N$ can be generated by elements of order 2 then $N \subseteq Z(G)$.

\section{A Characterization}

Observe that $C_{3}$ is a quotient of $A_{4}$ and $\alpha\left(C_{3}\right)=\alpha\left(A_{4}\right)=2 / 3$, so it is not always the case that $\alpha(G)=\alpha(G / N)$ implies $G \cong N \times G / N$. We can characterize the groups such that $\alpha(G)=\alpha(G / N)$ when $G / N$ is a symmetric group, for the following two reasons: the symmetric groups can be generated by elements of order 2 and their double covers are known.

Theorem 1. Let $G$ be a group and $N$ a normal subgroup of $G$ such that $G / N$ is isomorphic to a symmetric group. If $\alpha(G)=\alpha(G / N)$ then $N$ is an elementary abelian 2-group and it admits a normal complement in $G$, so that $G \cong N \times G / N$.

Proof. We prove the result by induction on the order of $G$. By 2.4, $N$ is an elementary abelian 2 -group. Since $G / N \cong S_{m}$ can be generated by elements of order 2, 2.8 implies that $N$ is central in $G$. If $m=2$ then the result follows from 2.2 so suppose $m \geq 3$. Let $R \cong C_{2}{ }^{l}$ be a minimal normal subgroup of $G$ contained in $N$. By 2.6 we have $\alpha(G / R)=\alpha(G)=\alpha\left(S_{m}\right)$ so by induction, since $G / N$ is a quotient of $G / R$, we have $G / R=C_{2}^{l} \times S_{m}$ for some $l \geq 0$. Let $K \unlhd G$ be the (normal) subgroup of $G$ such that $K / R=\{1\} \times S_{m}$. Observe that $K \cap N$ contains $R$, so $K \cap N / R$ is a normal 2-subgroup of $K / R \cong S_{m}$. If $m \neq 4$ this implies that $K \cap N=R$ because $S_{m}$ in this case does not admit non-trivial normal 2-subgroups (being $m \geq 3$ ). If $m=4$ and $K \cap N \neq R$ then $K \cap N / R$ is the Klein group, and $K / K \cap N \cong S_{3}$. However in this case 2.3 and 2.7 imply that

$$
17 / 24=\alpha\left(S_{4}\right)=\alpha(K / R) \geq \alpha(K)=\alpha(K / K \cap N)=\alpha\left(S_{3}\right)=5 / 6,
$$

a contradiction. We deduce that $K \cap N=R$.

If $N \neq R$ then $|K|<|G|$ and $\alpha(K)=\alpha(K / R)=\alpha\left(S_{m}\right)$ by 2.7 (being $K \cap N=$ $R)$. By induction we deduce that $K \cong R \times S_{m}$. Set $M:=\{1\} \times S_{m} \leq K$. Since

$$
S_{m} \cong G / N \geq K N / N \cong K / K \cap N=K / R \cong S_{m}
$$


we obtain $G=K N=M R N=M N$ so being $N$ central in $G$ and $N \cap M=$ $N \cap K \cap M=R \cap M=\{1\}$ we deduce $G=N \times M \cong N \times G / N$. Assume now $N=R$, so $N$ is a minimal normal subgroup of $G$. Since $N$ is central, $|N|=2$ and actually $N=\langle z\rangle=Z(G)$ is the center of $G$ (being $G / N \cong S_{m}$ with $m \geq 3$ ). Suppose by contradiction that $G$ is not a direct product $C_{2} \times S_{m}$. We claim that $N$ is contained in the derived subgroup of $G$. Indeed $G^{\prime}$ is contained in the subgroup $T$ of $G$ such that $T \supseteq N, T / N \cong A_{m}$ (being $|G / T|=2$ ), so if $G^{\prime}$ does not contain $N$ then $G^{\prime} N / N$ is a nontrivial normal subgroup of $G / N \cong S_{m}$ containing the derived subgroup of $S_{m}$ (that is, $A_{m}$ ) hence $G^{\prime} N=T$ therefore letting $\varepsilon \in G$ represent a fixed element of order 2 of $G / N \cong S_{m}$ not belonging to $A_{m}, \varphi(o(\varepsilon))=\varphi(2)=1$ (by [2.3) hence $o(\varepsilon)=2$ implying that $G^{\prime}\langle\varepsilon\rangle \cap N=\{1\}$ (otherwise $G^{\prime}\langle\varepsilon\rangle \supseteq N$ implying that $G^{\prime}\langle\varepsilon\rangle=G$ so $\left|G: G^{\prime}\right|=2$ hence $G \cong N \times G^{\prime}$, a contradiction) therefore $G^{\prime}\langle\varepsilon\rangle \cong G^{\prime}\langle\varepsilon\rangle N / N=G / N \cong S_{m}$; being $N$ the center of $G$ we deduce $G \cong C_{2} \times S_{m}$, a contradiction. This implies that $N \subseteq G^{\prime}$ so $G$ is a double cover of $S_{m}$ (that is, a stem extension of $S_{m}$ where the base normal subgroup has order 2 ), and looking at the known presentations of the double covers of the symmetric group (classified by Schur, see for example [9]) we see that $z$ is a square in $G$, that is there exists $x \in G$ with $x^{2}=z$, so that $x$ has order 4 and $x N$ has order 2, contradicting $\varphi(o(x))=\varphi(o(x N))$ (which is true by 2.3).

\section{NiLPOTENT GROUPS}

Let $G$ be a finite group. For $\ell$ a divisor of $|G|$ let $B_{G}(\ell)$ be the number of elements $g \in G$ with the property that $g^{\ell}=1$ and let $r_{G}(\ell)$ be the number of elements of $G$ of order $\ell$. It is worth mentioning the famous result by Frobenius that if $G$ is any group and $\ell$ divides $|G|$ then $\ell$ divides $B_{G}(\ell)$. The idea of the following result, which is a reformulation of formula (1) in the nilpotent case, is to give a formula for $c(G)$ when $G$ is a nilpotent group in terms of the numbers $B_{G}(d)$, that in general are reasonably easy to deal with (consider for example the case in which $G$ is abelian).

Theorem 2. If $G$ is a nilpotent group of order $n$ then $c(G)=\sum_{d \mid n} B_{G}(d) / d$.

Proof. Assume first that $G$ is a $p$-group, $|G|=p^{n}$. Since $r_{G}\left(p^{j}\right)=B_{G}\left(p^{j}\right)-$ $B_{G}\left(p^{j-1}\right)$ whenever $j \geq 1$ we see that

$$
\begin{aligned}
c(G) & =\sum_{x \in G} \frac{1}{\varphi(o(x))}=\sum_{j=0}^{n} \frac{r_{G}\left(p^{j}\right)}{\varphi\left(p^{j}\right)}=1+\sum_{j=1}^{n} \frac{B_{G}\left(p^{j}\right)-B_{G}\left(p^{j-1}\right)}{\varphi\left(p^{j}\right)} \\
& =1-\frac{B_{G}(1)}{\varphi(p)}+\sum_{j=1}^{n-1}\left(\frac{1}{\varphi\left(p^{j}\right)}-\frac{1}{\varphi\left(p^{j+1}\right)}\right) B_{G}\left(p^{j}\right)+\frac{B_{G}\left(p^{n}\right)}{\varphi\left(p^{n}\right)}=\sum_{j=0}^{n} \frac{B_{G}\left(p^{j}\right)}{p^{j}} .
\end{aligned}
$$

Now consider the general case, and write the order of $G$ as $n=|G|=p_{1}{ }^{n_{1}} \cdots p_{t}{ }^{n_{t}}$ with the $p_{i}$ 's pairwise distinct primes, $G$ is a direct product $\prod_{i=1}^{t} G_{p_{i}}$ where $G_{p_{i}}$ is the unique Sylow $p_{i}$-subgroup of $G$. Using 2.1 we obtain

$$
c(G)=\prod_{i=1}^{t} c\left(G_{p_{i}}\right)=\prod_{i=1}^{t}\left(\sum_{j=0}^{n} \frac{B_{G}\left(p_{i}^{j}\right)}{p_{i}^{j}}\right)=\sum_{d \mid n} B_{G}(d) / d .
$$

The last equality follows from the fact that since $G$ is nilpotent $B_{G}(a b)$ equals $B_{G}(a) B_{G}(b)$ if $a$ and $b$ are coprime divisors of $n$. 


\section{An ASYMPtotic RESUlT}

We want to study $\alpha\left(G^{n}\right)$ where $G^{n}=G \times G \times \cdots \times G$ ( $n$ times) in terms of the functions $B_{G}$ and $r_{G}$ defined in the previous section. Recall that the exponent of a group $G$, denoted $\exp (G)$, is the least common multiple of the orders of the elements of $G$. It is clear that $\exp \left(G^{n}\right)=\exp (G)$. The following result shows that $G^{n}$ has roughly $\left|G^{n}\right| / \varphi(\exp (G))$ cyclic subgroups.

Theorem 3. Let $G$ be a finite group. Then $\lim _{n \rightarrow \infty} \alpha\left(G^{n}\right)=1 / \varphi(\exp (G))$.

Proof. Observe that $r_{G^{n}}(\ell) \neq 0$ only if $\ell$ divides $|G|$, therefore

$$
\alpha\left(G^{n}\right)=\frac{1}{\left|G^{n}\right|} \sum_{x \in G^{n}} \frac{1}{\varphi(o(x))}=\sum_{\left.\ell|| G\right|^{n}} \frac{r_{G^{n}}(\ell)}{\varphi(\ell)|G|^{n}}=\sum_{\ell|| G \mid} \frac{r_{G^{n}}(\ell)}{\varphi(\ell)|G|^{n}}
$$

so what we need to compute is the limit $L_{\ell}$ of $r_{G^{n}}(\ell) /|G|^{n}$ when $n \rightarrow \infty$, for $\ell$ a divisor of $|G|$. Clearly $r_{G^{n}}(\ell) \leq B_{G^{n}}(\ell)=B_{G}(\ell)^{n}$ hence $r_{G^{n}}(\ell) /|G|^{n} \leq\left(B_{G}(\ell) /|G|\right)^{n}$ so if $B_{G}(\ell)<|G|$ then $L_{\ell}=0$. Now assume $B_{G}(\ell)=|G|$, in other words $\exp (G)$ divides $\ell$. If $\exp (G)<\ell$ then $r_{G^{n}}(\ell)=0$ so $L_{\ell}=0$. Now assume $\exp (G)=\ell$. Let $p$ vary in the set of prime divisors of $|G|$, and for every such $p$ define $a_{p}:=B_{G}(\exp (G) / p)$. Clearly $G^{n}$ has at least $|G|^{n}-\sum_{p} a_{p}{ }^{n}$ elements of order $\exp (G)$. Observe that $a_{p}<|G|$ by definition of $\exp (G)$, so that $a_{p} /|G|<1$, hence $\left(a_{p} /|G|\right)^{n}$ tends to 0 as $n \rightarrow \infty$, implying $L_{\exp (G)}=1$. The result follows.

\section{A CONNECTION With the PRobability of COMMUtation}

The probability that two elements in a group $G$ commute is denoted by $\operatorname{cp}(G)$ ("commuting probability" of $G$ ) and is defined by $|S| /|G \times G|$ where $S$ is the set of pairs $(x, y) \in G \times G$ such that $x y=y x$. It is easy to show that $\operatorname{cp}(G)=k(G) /|G|$ where $k(G)$ is the number of conjugacy classes of $G$. This invariant was studied by many authors, but we refer mostly to $[5]$.

Let $I(G)$ be the size of the set $\left\{x \in G: x^{2}=1\right\}$. The following lemma is easily deducible from Theorem 2J of [1]. It can also be proved character-theoretically using the Frobenius-Schur indicator.

Lemma 1. $I(G)^{2} \leq k(G)|G|$, in other words $\operatorname{cp}(G) \geq(I(G) /|G|)^{2}$.

This together with 2.2 implies the following inequality.

Lemma 2. If $\alpha(G) \geq 1 / 2$ then $\operatorname{cp}(G) \geq(2 \alpha(G)-1)^{2}$.

Let us include some other results from [5] that we will need in the following section.

6.1. If $G$ is a non-solvable group and $\operatorname{sol}(G)$ is the maximal normal solvable subgroup of $G$ then $\operatorname{cp}(G) \leq|G: \operatorname{sol}(G)|^{-1 / 2}$. This follows from [5, Theorem 9] (which depends on the classification of the finite simple groups), and together with Lemma 2 implies that if $\alpha(G)>1 / 2$ then $|G: \operatorname{sol}(G)| \leq(2 \alpha(G)-1)^{-4}$.

6.2. If $G$ is a solvable group and $F(G)$ is the Fitting subgroup of $G$ then $\operatorname{cp}(G) \leq$ $|G: F(G)|^{-1 / 2}$. This follows from [5, Theorem 4], and together with Lemma 2 implies that if $\alpha(G)>1 / 2$ then $|G: F(G)| \leq(2 \alpha(G)-1)^{-4}$. 


\begin{tabular}{|l|l|l|l|l|}
\hline$G$ & $\operatorname{Aut}(G)$ & $|G|$ & Float $(\alpha(G))$ & $\alpha(G)$ \\
\hline $\operatorname{Alt}(5)$ & $\operatorname{Sym}(5)$ & 60 & 0.533333 & $8 / 15$ \\
\hline $\operatorname{Sym}(5)$ & $\operatorname{Sym}(5)$ & 120 & 0.558333 & $67 / 120$ \\
\hline $\operatorname{Alt}(6)$ & $\operatorname{P\Gamma L}(2,9)$ & 360 & 0.463889 & $167 / 360$ \\
\hline $\operatorname{PGL}(2,9)$ & $\operatorname{P\Gamma L}(2,9)$ & 720 & 0.394444 & $71 / 180$ \\
\hline $\operatorname{Sym}(6)$ & $\operatorname{P\Gamma L}(2,9)$ & 720 & 0.502778 & $181 / 360$ \\
\hline $\mathrm{M}_{10}$ & $\operatorname{P\Gamma L}(2,9)$ & 720 & 0.419444 & $151 / 360$ \\
\hline $\operatorname{P\Gamma L}(2,9)$ & $\operatorname{P\Gamma L}(2,9)$ & 1440 & 0.426389 & $307 / 720$ \\
\hline $\operatorname{Alt}(7)$ & $\operatorname{Sym}(7)$ & 2520 & 0.375794 & $947 / 2520$ \\
\hline $\operatorname{Sym}(7)$ & $\operatorname{Sym}(7)$ & 5040 & 0.404563 & $2039 / 5040$ \\
\hline $\operatorname{PSL}(3,2)$ & $\operatorname{PGL}(2,7)$ & 168 & 0.470238 & $79 / 168$ \\
\hline $\operatorname{PGL}(2,7)$ & $\operatorname{PGL}(2,7)$ & 336 & 0.464286 & $13 / 28$ \\
\hline $\operatorname{PSL}(2,8)$ & $\operatorname{P\Gamma L}(2,8)$ & 504 & 0.309524 & $13 / 42$ \\
\hline $\operatorname{P\Gamma L}(2,8)$ & $\operatorname{P\Gamma L}(2,8)$ & 1512 & 0.362434 & $137 / 378$ \\
\hline $\operatorname{PSL}(2,11)$ & $\operatorname{PGL}(2,11)$ & 660 & 0.369697 & $61 / 165$ \\
\hline $\operatorname{PGL}(2,11)$ & $\operatorname{PGL}(2,11)$ & 1320 & 0.368182 & $81 / 220$ \\
\hline $\operatorname{PSL}(2,13)$ & $\operatorname{PGL}(2,13)$ & 1092 & 0.335165 & $61 / 182$ \\
\hline $\operatorname{PGL}(2,13)$ & $\operatorname{PGL}(2,13)$ & 2184 & 0.322344 & $88 / 273$ \\
\hline $\operatorname{PSL}(2,17)$ & $\operatorname{PGL}(2,17)$ & 2448 & 0.306373 & $125 / 408$ \\
\hline $\operatorname{PGL}(2,17)$ & $\operatorname{PGL}(2,17)$ & 4896 & 0.26777 & $437 / 1632$ \\
\hline $\operatorname{PSL}(2,19)$ & $\operatorname{PGL}(2,19)$ & 3420 & 0.267251 & $457 / 1710$ \\
\hline $\operatorname{PSL}(2,16)$ & $\operatorname{P\Gamma L}(2,16)$ & 4080 & 0.192157 & $49 / 255$ \\
\hline
\end{tabular}

TABLE 1. Almost-simple groups of order at most 5397

\section{NON-SOLVABLE GROUPS}

Theorem 4. Let $G$ be a finite non-solvable group. Then $\alpha(G) \leq \alpha\left(S_{5}\right)$ with equality if and only if $G \cong S_{5} \times C_{2}{ }^{n}$ for some integer $n \geq 0$.

Proof. Let $\alpha:=\alpha\left(S_{5}\right)=67 / 120$. We will show that if $G$ is any finite nonsolvable group such that $\alpha(G) \geq \alpha$ then $G \cong S_{5} \times C_{2}{ }^{n}$ for some $n \geq 0$. Assume $\alpha(G) \geq \alpha$, in particular $\alpha(G)>1 / 2$. By 6.1 we deduce $|G / \operatorname{sol}(G)| \leq$ $(2 \alpha-1)^{-4}=(60 / 7)^{4}<5398$ thus $|G / \operatorname{sol}(G)| \leq 5397$. Observe that $G / \operatorname{sol}(G)$ is non-trivial (being $G$ non-solvable), it does not have non-trivial solvable normal subgroups and $\alpha(G / \operatorname{sol}(G)) \geq \alpha(G) \geq \alpha$. If we can show that $G / \operatorname{sol}(G) \cong S_{5}$ it will follow that $67 / 120=\alpha \leq \alpha(G) \leq \alpha(G / \operatorname{sol}(G))=\alpha\left(S_{5}\right)=67 / 120$ therefore $\alpha(G)=\alpha(G / \operatorname{sol}(G))$ and the result follows from Theorem 1 .

We are left to show that if $G$ is a group without non-trivial solvable normal subgroups and such that $|G| \leq 5397$ and $\alpha(G) \geq \alpha\left(S_{5}\right)=67 / 120$, then $G \cong$ $S_{5}$. Let $N$ be a minimal normal subgroup of $G$, then $N=S^{t}$ with $S$ a nonabelian simple group. If $t \geq 2$ then being $|S| \geq 60$ and $|G| \leq 5397$ we deduce $G=N=A_{5} \times A_{5}$, contradicting the minimality of $N$. So $t=1$. We claim that there is no other minimal normal subgroup of $G$. Indeed if $M$ is a minimal normal subgroup of $G$ distinct from $N$ then $M$ is non-solvable (by assumption) so $|G: M N|=|G| /|M N| \leq 5397 / 60^{2}<2$ (the smallest order of a non-solvable group is 60) so $G=M N$ and actually $G=M \times N=A_{5} \times A_{5}$ ( $M$ is a direct power of a non-abelian simple group, the smallest orders of non-abelian simple groups are 60 , 168 and $60 \cdot 60^{2}, 60 \cdot 168$ are both larger than 5397) which is a contradiction because 
$\alpha\left(A_{5} \times A_{5}\right)=77 / 225<\alpha\left(S_{5}\right)$. We deduce that $N$ is the unique minimal normal subgroup of $G$. Since $N$ is non-solvable, it is non-abelian, so it is not contained in $C_{G}(N)$, hence $C_{G}(N)$ must be trivial (otherwise it would contain a minimal normal subgroup of $G$ distinct from $N$ ) therefore $G$ is almost-simple. Using 7 and [3] we computed the list of almost-simple groups $G$ of size at most 5397 and for each of them we determined $\alpha(G)$. The results are summarized in the above table. We deduce that the only almost-simple group $G$ with $|G| \leq 5397$ and $\alpha(G) \geq 67 / 120$ is $G=S_{5}$.

This together with Lemma 2 and 6.2 implies the following.

Corollary 1. If $\alpha(G)>\alpha\left(S_{5}\right)$ then $G$ is solvable and the Fitting subgroup of $G$ has index at most 5397.

\section{Groups With MANY CYCLIC SUbGROUPS}

In this section we will study groups with $\alpha(G)$ "large", specifically, we will classify all the finite groups $G$ such that $\alpha(G)>3 / 4$. This is a natural choice because $3 / 4$ turns out to be the largest non-trivial accumulation point of the set of numbers of the form $\alpha(G)$. To do such classification the idea is to observe that if $\alpha(G)>3 / 4$ then $I(G) /|G|>1 / 2$ and use Wall's classification [10, Section 7].

Theorem 5. Let $X$ be a group with $\alpha(X)>3 / 4$. Then $X$ is a direct product of an elementary abelian 2-group with a group $G, \alpha(X)=\alpha(G), G$ does not have $C_{2}$ as a direct factor, and either $G$ is trivial (in which case $\alpha(X)=1$ ) or one of the following occurs.

(1) Case I. $G \cong A \rtimes\langle\varepsilon\rangle$, where $\langle\varepsilon\rangle=C_{2}$ acts on $A$ by inversion and there exists an integer $n \geq 1$ such that one of the following occurs.

$$
A=C_{3}{ }^{n}, \alpha(G)=\frac{3 \cdot 3^{n}+1}{4 \cdot 3^{n}} \quad \text { or } \quad A=C_{4}{ }^{n}, \alpha(G)=\frac{3 \cdot 2^{n}+1}{4 \cdot 2^{n}} .
$$

(2) Case II. $G \cong D_{8} \times D_{8}$ and $\alpha(G)=25 / 32$.

(3) Case III. $G$ is a quotient $D_{8}{ }^{r} / N$ where $N=\left\{\left(a_{1}, \ldots, a_{r}\right) \in Z\left(D_{8}\right)^{r}\right.$ : $\left.a_{1} \cdots a_{r}=1\right\}$ and

$$
\alpha(G)=\frac{3 \cdot 2^{r}+1}{4 \cdot 2^{r}}
$$

(4) Case IV. G is a semidirect product $V \rtimes\langle c\rangle$ where $V=\mathbb{F}_{2}{ }^{2 r}$ has a basis $\left\{x_{1}, y_{1}, \ldots, x_{r}, y_{r}\right\}, c$ has order 2 , it acts trivially on each $y_{i}, x_{i}^{c}=c x_{i} c=$ $\left[c, x_{i}\right] x_{i}=x_{i} y_{i}$ for $i=1, \ldots, r$, and

$$
\alpha(G)=\frac{3 \cdot 2^{r}+1}{4 \cdot 2^{r}}
$$

Proof. We know by 2.5 that $\alpha(X)=\alpha(G)$. Also, we may assume that $G$ is nontrivial. Since $\alpha(G)>3 / 4$, by 2.2 we have $I(G) /|G| \geq 2 \alpha(G)-1>1 / 2$ so $G$ appears in Wall's classification [10, Section 7]. Case II is immediate, we will treat cases I, III and IV.

Case I of Wall's classification. $G$ is a semidirect product $A \rtimes\langle\varepsilon\rangle$ with $A$ an abelian group, $\langle\varepsilon\rangle \cong C_{2}$ and every element of $G-A$ has order 2 . Observe that $A$ does not admit $C_{2}$ as a direct factor. Indeed if $a \in A$ then since $a \varepsilon \notin A$, $a \varepsilon$ has order 2 so $a^{\varepsilon}=a^{-1}$, hence $\varepsilon$ acts on $A$ as inversion and a direct factor of order 2 in $A$ would yield a direct factor of order 2 in $G$. It follows that $c(G)=c(A)+|G| / 2$, 
so that $3 / 4<\alpha(G)=\alpha(A) / 2+1 / 2$ implying $\alpha(A)>1 / 2$. If the prime $p$ divides the order of $A$ then $C_{p}$ is a quotient of $A$ so $1 / 2<\alpha(A) \leq \alpha\left(C_{p}\right)=2 / p$ whence $p \leq 3$, that is, $p$ is either 2 or 3. Write $A=P_{2} \times P_{3}$ where $P_{2}$ is an abelian 2-group and $P_{3}$ is an abelian 3-group. Observe that $C_{9}$ is not a quotient of $A$ because otherwise $1 / 2<\alpha(A) \leq \alpha\left(C_{9}\right)=1 / 3$, a contradiction. Therefore if $P_{2}$ is trivial then $A=C_{3}{ }^{n}$ for some $n \geq 1$, an easy computation shows $\alpha(A)=\frac{3^{n}+1}{2 \cdot 3^{n}}$, and the result follows. Suppose now that $P_{2}$ is non-trivial. If $P_{3}$ is non-trivial then since $P_{2}$ is not elementary abelian (because $A$ does not have $C_{2}$ as a direct factor) there is a quotient of $A$ isomorphic to $C_{12}$, however $1 / 2<\alpha(A) \leq \alpha\left(C_{12}\right)=1 / 2$ gives a contradiction. So $P_{3}=\{1\}$, in other words $A$ is an abelian 2-group and we may write $A=\prod_{i=1}^{n} C_{2^{a_{i}}}$. Since $A$ does not have $C_{2}$ as a direct factor we deduce $a_{i} \geq 2$ for all $i$, on the other hand if one of the $a_{i}$ 's is at least 3 then $C_{8}$ is a quotient of $A$ but $1 / 2<\alpha(A) \leq \alpha\left(C_{8}\right)=1 / 2$ is a contradiction. So $A \cong C_{4}{ }^{n}$ hence an easy computation shows $\alpha(A)=\frac{2^{n}+1}{2 \cdot 2^{n}}$, and the result follows.

Case III of Wall's classification. $G$ is a direct product of $D_{8}$ 's with the centers amalgamated. $G=G(r)$ has a presentation

$$
G(r)=\left\langle c, x_{1}, y_{1}, \ldots, x_{r}, y_{r}: c^{2}=x_{i}^{2}=y_{i}^{2}=1,\right.
$$

all pairs of generators commute except $\left.\left[x_{i}, y_{i}\right]=c\right\rangle$.

A more practical description of the group in question is $G=D_{8}{ }^{r} / N$ where $N=$ $\left\{\left(z_{1}, \ldots, z_{r}\right) \in Z^{r}: z_{1} \cdots z_{r}=1\right\}$ where $Z=\langle z\rangle$ (cyclic of order 2 ) is the center of $D_{8} . \quad N$ is a subgroup of $Z\left(D_{8}{ }^{r}\right)=Z^{r}$ of index 2 , so $|G|=2 \cdot 4^{r}$. An element $\left(a_{1}, \ldots, a_{r}\right) N \in G$ squares to 1 if and only if $\left(a_{1}^{2}, \ldots, a_{r}^{2}\right) \in N$, that is, $a_{1}^{2} \cdots a_{r}^{2}=1$. Observe that every $a_{i}^{2}$ is either 1 or $z$, so this condition means that there are an even number of indeces $i$ such that $a_{i}^{2}=z$. Since $D_{8}$ contains 6 elements that square to 1 and 2 elements that square to $z, D_{8}{ }^{r}$ contains exactly

$$
\beta_{r}=\sum_{k=0}^{[r / 2]}\left(\begin{array}{c}
r \\
2 k
\end{array}\right) 2^{2 k} 6^{r-2 k}
$$

elements $\left(a_{1}, \ldots, a_{r}\right)$ such that $a_{1}^{2} \cdots a_{r}^{2}=1$. Hence $G$ contains exactly $\beta_{r} / 2^{r-1}$ elements that square to 1 . Observe that

$$
8^{r}=(2+6)^{r}=\sum_{h=0}^{r}\left(\begin{array}{l}
r \\
h
\end{array}\right) 2^{h} 6^{r-h}, \quad 4^{r}=(-2+6)^{r}=\sum_{h=0}^{r}\left(\begin{array}{l}
r \\
h
\end{array}\right)(-1)^{h} 2^{h} 6^{r-h}
$$

so adding them together gives exactly $2 \beta_{r}$. This means that $\beta_{r}=\frac{1}{2}\left(8^{r}+4^{r}\right)$, so $G$ has exactly $\beta_{r} / 2^{r-1}=4^{r}+2^{r}$ elements that square to 1 and exactly $|G|-\beta_{r} / 2^{r-1}=$ $2 \cdot 4^{r}-4^{r}-2^{r}=4^{r}-2^{r}$ elements of order 4 . Therefore

$$
\alpha(G)=\frac{1}{2 \cdot 4^{r}}\left(4^{r}+2^{r}+\frac{1}{2}\left(4^{r}-2^{r}\right)\right)=\frac{3 \cdot 2^{r}+1}{4 \cdot 2^{r}} .
$$

Case IV of Wall's Classification. $G=G(r)$ has a presentation

$$
G(r)=\left\langle c, x_{1}, y_{1}, \ldots, x_{r}, y_{r}: c^{2}=x_{i}^{2}=y_{i}^{2}=1,\right.
$$

all pairs of generators commute except $\left.\left[c, x_{i}\right]=y_{i}\right\rangle$.

A more practical description of the group in question is $G \cong V \rtimes\langle c\rangle$ where $V=$ $C_{2}{ }^{2 r}=\left\langle x_{1}, y_{1}, \ldots, x_{r}, y_{r}\right\rangle, c$ has order 2 , it acts trivially on each $y_{i}$ and $x_{i}^{c}=c x_{i} c=$ $\left[c, x_{i}\right] x_{i}=x_{i} y_{i}$ for $i=1, \ldots, r$. Thinking of $V$ as a vector space over $\mathbb{F}_{2}$, if $v \in V$ 
then $v c$ has order 2 or 4 , and it has order 4 exactly when $v^{c} \neq v$. Observe that with respect to the given basis of $V$ the operator $c$ (acting from the right) has a diagonal block matrix form with $J=\left(\begin{array}{ll}1 & 1 \\ 0 & 1\end{array}\right)$ on each diagonal block entry. Thus there are precisely $2^{r}$ vectors $v$ with $v^{c}=v$, they are of the form $\left(0, b_{1}, 0, b_{2}, \ldots, 0, b_{r}\right)$. Therefore $G$ has $2^{2 r}-2^{r}$ elements of order 4 and $c(G)=2^{2 r}+2^{r}+\left(2^{2 r}-2^{r}\right) / 2$. This implies that $\alpha(G)=\frac{3 \cdot 2^{r}+1}{4 \cdot 2^{r}}$.

The following corollary is immediate. It implies that if $G$ is a non-nilpotent group then $\alpha(G) \leq 5 / 6$ with equality if and only if $G$ is a direct product $C_{2}{ }^{n} \times S_{3}$.

Corollary 2. Let $G$ be a group such that $\alpha(G) \geq 5 / 6=\alpha\left(S_{3}\right)$. Then either

(1) $G \cong C_{2}{ }^{n} \times S_{3}$ for some $n \geq 0$ and $\alpha(G)=5 / 6$, or

(2) $G \cong C_{2}{ }^{n} \times D_{8}$ for some $n \geq 0$ and $\alpha(G)=7 / 8$, or

(3) $G \cong C_{2}{ }^{n}$ for some $n \geq 0$ and $\alpha(G)=1$.

We can deduce a bound of $|G|$ in terms of $|G|-c(G)$. The inequality $\alpha(G) \leq 7 / 8$ can be written as $|G| \leq 8(|G|-c(G))$, so we obtain the following.

Corollary 3. If $G$ is any finite group which is not an elementary abelian 2-group then $|G| \leq 8(|G|-c(G))$ with equality if and only if $G \cong D_{8} \times C_{2}{ }^{n}$ for some non-negative integer $n$.

Observe that the above results extend and generalize the results in [11. As an example of application let us determine the groups $G$ with $|G|-9$ cyclic subgroups. In this case we have $|G|-c(G)=9$ so $|G| \leq 72$ and a GAP check yields that $G$ is one of $C_{11}, D_{22}$ and $C_{4} \times S_{3}$.

\section{SPeCial FAMILIES OF GROUPS}

Proposition 1. Let $p \geq 3$ be a prime number. Let $G$ be a non-trivial group of order divisible only by primes at least $p$. Then $\alpha(G) \leq 2 / p$ with equality if and only if $G \cong C_{p}$.

In particular if $G$ belongs to the family of groups of odd order then $\alpha(G) \leq 2 / 3$ with equality if and only if $G \cong C_{3}$.

Proof. If $1 \neq x \in G$ and $q$ is a prime divisor of the order of $x$ then $p \leq q$ so $\varphi(o(x)) \geq \varphi(q)=q-1 \geq p-1$, so since $|G| \geq p$ we have

$$
\begin{aligned}
\alpha(G) & =\frac{1}{|G|} \sum_{x \in G} \frac{1}{\varphi(o(x))} \leq \frac{1}{|G|}\left(1+\frac{|G|-1}{p-1}\right) \\
& =\frac{p-2}{|G|(p-1)}+\frac{1}{p-1} \leq \frac{p-2}{p(p-1)}+\frac{1}{p-1}=\frac{2}{p} .
\end{aligned}
$$

If equality holds the above inequalities are equalities and using $p \geq 3$ it is easy to deduce that $|G|=p$, that is, $G \cong C_{p}$.

Proposition 2. Let $G$ be a group and $p$ an odd prime, and suppose $G$ has $C_{p}$ as epimorphic image (in other words $p$ divides $\left|G / G^{\prime}\right|$ ). Then $\alpha(G) \leq 2 / p$ with equality if and only if $G$ is a direct product of an elementary abelian 2-group with a Frobenius group with 2-elementary abelian kernel and complements of order $p$.

Observe that a bound of $2 / p$ when $p=2$ would be trivial. This is why we are only considering the odd case. 
Proof. Since $C_{p}$ is a quotient of $G$ we have $\alpha(G) \leq \alpha\left(C_{p}\right)=2 / p$. Now assume equality holds. Then $\alpha(G)=\alpha\left(C_{p}\right)$ and there exists $N \unlhd G$ with $G / N \cong C_{p}$, so $N$ is an elementary abelian 2-group by 2.4, say $N \cong C_{2}{ }^{m}$. If $G$ has elements of order $2 p$ then it has $C_{2}$ as a direct factor (for example by Maschke's theorem), so now assume $G$ does not have elements of order $2 p$. A subgroup of $G$ of order $p$ acts fixed point freely on $N$ so $G$ is a Frobenius group with Frobenius kernel equal to $N$ and Frobenius complement of order $p$. Now assume $G$ is a Frobenius group with 2 -elementary abelian kernel of size $2^{m}$ and Frobenius complement of order $p$. The element orders of $G$ are 1,2 , and $p$, and $G$ has precisely $2^{m}-1$ elements of order 2 and $2^{m}(p-1)$ elements of order $p$. We have

$$
\alpha(G)=\frac{1}{|G|} \sum_{x \in G} \frac{1}{\varphi(o(x))}=\frac{1}{2^{m} \cdot p}\left(2^{m}+\frac{2^{m}(p-1)}{p-1}\right)=2 / p .
$$

This concludes the proof.

\section{NON-SUPERSOLVABLE GROUPS}

Let $G$ be a solvable group and let $F_{i}$ be normal subgroups of $G$ defined as follows:

$$
F_{0}=\{1\}, \quad F_{i+1} / F_{i}:=F\left(G / F_{i}\right) \quad \forall i \geq 0,
$$

where $F\left(G / F_{i}\right)$ denotes the Fitting subgroup of $G / F_{i}$. In particular $F_{1}$ is the Fitting subgroup of $G$. Since $G$ is solvable, there exists a minimal $h$ such that $F_{h}=G$, such $h$ is called the "Fitting height" of $G$. Observe that

$$
F\left(F_{l} / F_{i-1}\right)=F_{i} / F_{i-1} \quad \forall l \geq i \geq 1,
$$

indeed $F_{i} / F_{i-1}$ is nilpotent and normal in $F_{l} / F_{i-1}$ hence $F_{i} / F_{i-1} \subseteq F\left(F_{l} / F_{i-1}\right)$, and $F\left(F_{l} / F_{i-1}\right)$ is nilpotent and characteristic in $F_{l} / F_{i-1}$, which is normal in $G / F_{i-1}$, so $F\left(F_{l} / F_{i-1}\right)$ is normal in $G / F_{i-1}$ hence $F\left(F_{l} / F_{i-1}\right) \subseteq F\left(G / F_{i-1}\right)=$ $F_{i} / F_{i-1}$. This implies in particular that $F_{l} / F_{i}$ has Fitting height $l-i$.

The following consequence of the solution of the $k(G V)$ problem is proved in 5 , Lemma 3, proof of (i)] (see also [6]).

Proposition 3. Let $G$ be a group and let $F$ be the Fitting subgroup of $G$. If $G / F$ is nilpotent (that is, $G$ has Fitting height 2) then $k(G) \leq|F|$, so that

$$
\operatorname{cp}(G) \leq \frac{1}{|G: F|} .
$$

The following result shows that $S_{4}$ is a "maximal" non-supersolvable group in terms of $\alpha(G)$.

Theorem 6. Let $G$ be a group. If $G$ is not supersolvable then $\alpha(G) \leq \alpha\left(S_{4}\right)$ with equality if and only if $G \cong C_{2}{ }^{n} \times S_{4}$ for some non-negative integer $n$.

Proof. We prove that if $\alpha(G) \geq \alpha\left(S_{4}\right)$ and $G$ is not supersolvable then $G$ is isomorphic to $C_{2}{ }^{n} \times S_{4}$ for some non-negative integer $n$. We have $\alpha(G) \geq \alpha\left(S_{4}\right)=$ $17 / 24>67 / 120$ so $G$ is solvable by Theorem 4, so the Fitting subgroup $F$ of $G$ is non-trivial, and since $G$ is not supersolvable $G \neq F$. Since $2 / 3<17 / 24$, Proposition 1 implies that $G$ does not have non-trivial quotients of odd order. Also, since $17 / 24>1 / 2$ we have $\operatorname{cp}(G) \geq(2 \alpha(G)-1)^{2} \geq 25 / 144$ by Lemma 2 . 
In the following discussion we will use Proposition 3. the inequality $\operatorname{cp}(G) \leq$ $\operatorname{cp}(N) \cdot \operatorname{cp}(G / N)$ for $N \unlhd G$ (see [5, Lemma 1]) and the obvious fact that the commuting probability is always at most 1 . Let $F_{i}$ be the subgroups defined above and let $h$ be the Fitting height of $G$. We distinguish three cases.

(1) $h=2$. In this case $G=F_{2}>F_{1}>\{1\}$. We have that $G / F_{1}$ is nilpotent so $25 / 144 \leq \operatorname{cp}(G) \leq 1 /\left|G: F_{1}\right|$ so $\left|G: F_{1}\right| \leq 5$. However $\left|G: F_{1}\right| \notin\{3,5\}$ because $G$ does not have non-trivial quotients of odd order, so $G / F_{1}$ is one of $C_{2}, C_{4}$ and $C_{2} \times C_{2}$.

(2) $h=3$. In this case $G=F_{3}>F_{2}>F_{1}>\{1\}$. We have $25 / 144 \leq \operatorname{cp}(G) \leq$ $\operatorname{cp}\left(F_{2}\right) \leq 1 /\left|F_{2}: F_{1}\right|$ and $25 / 144 \leq \operatorname{cp}(G) \leq \operatorname{cp}\left(G / F_{1}\right) \leq 1 /\left|G: F_{2}\right|$ so $\left|F_{2}: F_{1}\right| \leq 5$ and $\left|G: F_{2}\right| \leq 5$. Also $G / F_{1}$ is not a group of prime power order (because it is not nilpotent) and $\left|G: F_{2}\right|$ is not 3 or 5 because $G$ does not have quotients of odd order. Therefore $G / F_{1}$ is a group of order 6,10 , 12 or 20 , its Fitting subgroup has order at most 5 and $\alpha\left(G / F_{1}\right) \geq \alpha(G) \geq$ $17 / 24$. We deduce $G / F_{1} \cong S_{3}$ by $[3$.

(3) $h \geq 4$. In this case $G \geq F_{4}>F_{3}>F_{2}>F_{1}>\{1\}$. We have

$$
\frac{25}{144} \leq \mathrm{cp}(G) \leq \mathrm{cp}\left(F_{2}\right) \cdot \operatorname{cp}\left(F_{4} / F_{2}\right) \leq \frac{1}{\left|F_{2}: F_{1}\right|} \cdot \frac{1}{\left|F_{4}: F_{3}\right|}
$$

so $\left|F_{2}: F_{1}\right| \cdot\left|F_{4}: F_{3}\right| \leq 5$ implying that $\left|F_{2}: F_{1}\right|=\left|F_{4}: F_{3}\right|=2$. But then $F\left(F_{3} / F_{1}\right)=F_{2} / F_{1} \subseteq Z\left(F_{3} / F_{1}\right)$ implying $F_{3} / F_{1}=F_{2} / F_{1}$ (because the Fitting subgroup contains its own centralizer), a contradiction.

We deduce that $G / F$ is one of $C_{2}, C_{4}, C_{2} \times C_{2}$ and $S_{3}$.

Since $G$ is not supersolvable there exists a maximal subgroup $M$ of $G$ whose index $|G: M|$ is not a prime number (see [8, 9.4.4]). Let $M_{G}$ the normal core of $M$ in $G$, that is, the intersection of the conjugates of $M$ in $G$. Let $X:=G / M_{G}$, $K:=M / M_{G}$, so that $|G: M|=|X: K|$. Then $\alpha\left(S_{4}\right) \leq \alpha(G) \leq \alpha(X)$. This implies that if $X \cong S_{4}$ then the result follows from Theorem 11. so all we have to prove is that $X \cong S_{4}$. The subgroup $M / M_{G}$ of $X$ is maximal and it has trivial normal core, so $X$ is a primitive solvable group. We will make use of the known structural properties of primitive solvable groups, see for example [2, Section 15 of Chapter A]. $X$ is a semidirect product $X=V \rtimes K$ with $V=C_{p}{ }^{n}, p$ a prime, $V$ is the unique minimal normal subgroup of $X$ and it equals the Fitting subgroup of $X$. Since $|V|=|X: K|$ is not a prime, $n \geq 2$, so $|X|>6 \geq|G / F|$ hence $F \nsubseteq M_{G}$ so $F M_{G} / M_{G}$ is a non-trivial nilpotent normal subgroup of $X$ so it equals $V$, hence

$$
K \cong X / V=\left(G / M_{G}\right) /\left(F M_{G} / M_{G}\right) \cong G / F M_{G}
$$

is a quotient of $G / F$ so $K$ is one of $C_{2}, C_{4}, C_{2} \times C_{2}$ and $S_{3}$.

In what follows we will use the known representation theory of small groups over the field with $p$ elements. We will think of $V$ as a vector space of dimension $n$ over the field $\mathbb{F}_{p}$, irreducible when seen as a $\mathbb{F}_{p}[K]$-module. 
Suppose $p$ divides $|K|$. Observe that being not supersolvable, $X$ is not a 2-group, hence $K$ cannot be $C_{2}, C_{4}$ nor $C_{2} \times C_{2}$, so $K \cong S_{3}$. The structure of the group algebras $\mathbb{F}_{2}\left[S_{3}\right]$ and $\mathbb{F}_{3}\left[S_{3}\right]$ implies that $n \geq 2$ forces $n=p=2$ and hence $X \cong S_{4}$.

Suppose now $p$ does not divide $|K|$, so that $|K|$ and $|V|$ are coprime.

Suppose $\mathbb{F}_{p}$ is a splitting field for $K$. Since $n \geq 2$, the only possibility is $K \cong S_{3}$, $n=2$, and the action of $K$ on $V$ defining the group structure of $X=V \rtimes K$ is the following: $K \cong S_{3}$ permutes the coordinates of the vectors in the fully deleted module

$$
V=\left\{(a, b, c) \in \mathbb{F}_{p}^{3}: a+b+c=0\right\} .
$$

The elements of order 2 in $X$ are of the form $(a, b, c) k$ with $k \in K, o(k)=2$ and the fixed coordinate is zero, so there are $3 p$ of them. The elements of order 3 in $X$ are of the form $v k$ with $v \in V$ arbitrary and $k \in K, o(k)=3$, so there are $2 p^{2}$ of them. The elements of order $p$ in $X$ are the non-trivial elements of $V$ (being $p$ coprime to $|K|=6)$ so there are $p^{2}-1$ of them. The elements of order $2 p$ in $X$ are of the form $v k$ with $v \in V, k \in K, o(k)=2$ and $o(v k) \neq 2$ so there are $3 p^{2}-3 p$ of them. The exponent of $X$ is $6 p$ and $X$ has no elements of order $6,3 p$ or $6 p$. This implies that

$$
c(X)=\sum_{x \in X} \frac{1}{\varphi(o(x))}=1+3 p+\frac{2 p^{2}}{2}+\frac{p^{2}-1}{p-1}+\frac{3 p^{2}-3 p}{p-1}=p^{2}+7 p+2 .
$$

Using $p \geq 5$ and $|X|=6 p^{2}$ we deduce $\alpha(X)<17 / 24$, a contradiction.

Suppose $\mathbb{F}_{p}$ is not a splitting field for $K$. Since $n \geq 2$, the only possibility is $K \cong C_{4}, n=2$, and the polynomial $t^{2}+1$ does not split modulo $p$, that is, $p \equiv 3 \bmod 4$. Let $x$ be a generator of the cyclic group $K$. We may interpret $x$ as a matrix of order 4 , so that by irreducibility the minimal polynomial of $x$ is $t^{2}+1$ (the only irreducible factor of degree 2 of $t^{4}-1$ ). This implies that $x^{2}=-1$. Choosing a nonzero vector $v_{1} \in V$ and $v_{2}:=v_{1}^{x}$, since $v_{1}, v_{2}$ are linearly independent (otherwise $v_{1}$ would be an eigenvector for $x$ contradicting irreducibility), and $v_{2}{ }^{x}=v_{1}{ }^{2}=-v_{1}$, the matrix of $x$ in the base $\left\{v_{1}, v_{2}\right\}$ is $\left(\begin{array}{cc}0 & 1 \\ -1 & 0\end{array}\right)$ acting by right multiplication. $X=V \rtimes K$ has $p^{2}-1$ elements of order $p$ (the non-trivial elements of $V$, being $p$ coprime to $|K|=4$ ), $p^{2}$ elements of order 2 (the elements of the form $v x^{2}$ with $v \in V$ arbitrary) and $2 p^{2}$ elements of order 4 (the elements of the form $v x$ or $v x^{3}$ with $v \in V$ arbitrary). The exponent of $X$ is $4 p$ and there are no elements of order $2 p$ or $4 p$, therefore

$$
c(X)=\sum_{x \in X} \frac{1}{\varphi(o(x))}=1+p^{2}+\frac{2 p^{2}}{2}+\frac{p^{2}-1}{p-1}=2 p^{2}+p+2 .
$$

Using $p \geq 3$ and $|X|=4 p^{2}$ we deduce $\alpha(X)<17 / 24$, a contradiction.

\section{ACKnowledgements}

We would like to thank the referee for carefully reading a previous version of this paper and having very useful suggestions. 


\section{REFERENCES}

[1] R. Brauer, K. A. Fowler; On groups of even order. Ann. of Math. (2) 62 (1955), 565-583.

[2] K. Doerk and T. Hawkes, Finite Soluble Groups, de Gruyter Expositions in Mathematics 4, de Gruyter (1992).

[3] The GAP Group, GAP - Groups, Algorithms, and Programming, Version 4.8.

[4] Garonzi M., Patassini M.; Inequalities detecting structural properties of a finite group. Comm. Algebra 45 (2017), no. 2, 677-687.

[5] Guralnick R. M., Robinson G. R.; On the commuting probability in finite groups. J. Algebra 300 (2006), no. 2, 509-528.

[6] Knorr R.; On the number of characters in a $p$-block of a $p$-solvable group. Illinois J. Math. 28 (1984) 181-210.

[7] D. Leemans, L. Vauthier; An atlas of abstract regular polytopes for small groups. Aequationes Math. 72 (2006), no. 3, 313-320.

[8] D. J. S. Robinson, A course in the theory of groups; Second edition; Graduate Texts in Mathematics, 80. Springer-Verlag, New York, 1996.

[9] Schur, J; On the representation of the symmetric and alternating groups by fractional linear substitutions. Translated from the German [J. Reine Angew. Math. 139 (1911), 155-250] by Marc-Felix Otto. Internat. J. Theoret. Phys. 40 (2001), no. 1, 413-458.

[10] Wall, C. T. C.; On groups consisting mostly of involutions. Proc. Cambridge Philos. Soc. 67 $1970251-262$.

[11] Tarnauceanu, M.; Finite groups with a certain number of cyclic subgroups. Amer. Math. Monthly 122 (2015), no. 3, 275-276.

(Martino Garonzi) Departamento de Matemática, Universidade de Brasília, Campus Universitário Darcy Ribeiro, Brasília-DF, 70910-900, Brazil

E-mail address: mgaronzi@gmail.com

(Igor Lima) Universidade Federal de Goiás, imTec - Regional Catalão, Av. Dr. Lamartine P. de Avelar, 1120 Setor Universitário Catalão-GO, 75704-020, Brazil

E-mail address: igor.matematico@gmail.com 